\title{
II. „Que faire de l'Allemagne?"“: Die französische Deutschlandpolitik 1944-1949
}

„Das wesentliche Problem für Frankreich ist das Problem Deutschland. Das Problem Deutschland ist zugleich das europäische Problem par excellence. Als europäisches Problem par excellence ist das Problem in erster Linie ein globales Problem." Dieser Befund des Leiters der französischen Mission bei den Alliierten Regierungen in London Maurice Dejean wurde vom Chef der Provisorischen Regierung der Französischen Republik Charles de Gaulle allen Postulaten vom Primat der Ökonomie und der Innenpolitik nachgeborener Historiker zum Trotz dezidiert mitgetragen ${ }^{2}$. Folglich mußte es seiner Meinung nach darum gehen, Frankreich wieder den ihm zustehenden Rang in der Welt zu verschaffen und ihm einen Anteil beim Neuaufbau einer Friedensordnung und speziell bei den Diskussionen über die Zukunft Deutschlands zu sichern.

Wie kein anderer französischer Politiker der Nachkriegszeit verkörpert de Gaulle bis auf den heutigen Tag das Streben des Hexagons nach nationaler Größe und Weltgeltung. 1890 in Lille geboren, schlug er zunächst die Offizierslaufbahn ein, die ihn 1940 für wenige Wochen zum Unterstaatssekretär für Verteidigung im Generalsrang aufsteigen ließ. Nach dem Zusammenbruch gründete er in London das Komitee „France libre“ und leitete ab Juni 1943 als Président du Comité Français de Libération Nationale die Résistance. Nach der Befreiung übernahm er Anfang September 1944 den Vorsitz der aus diesem Comité hervorgehenden Provisorischen Regierung. Durch die Wahlen zur Assemblée Constituante im November $1945 \mathrm{zu}$ einer Mehrparteienregierung gezwungen, legte de Gaulle am 20.1. 1946 sein Amt nieder. 1947 gründete er eine Sammlungsbewegung nach seinem Gusto, das „Rassemblement du Peuple Français“, das er bis 1954 anführte, um sich anschließend auf seinen Landsitz Colombey-les-deux-Eglises zurückzuziehen. Erst die Bürgerkriegsgefahr des Mai 1958 sollte ihn in die aktive Politik zurückführen ${ }^{3}$.

Indem der General Frankreich nach dem Ende des Zweiten Weltkrieges wieder zu "grandeur" verhelfen und dem ihm seiner Ansicht nach zukommenden "rang“ als Großmacht zuführen wollte, orientierte er sich in seinen deutschlandpolitischen Zielen an den Vorstellungen traditioneller nationaler Machtpolitik ${ }^{4}$. So war die „campagne de l'Allemagne“ auch ein Symbol nationaler "résurrection“, wie

Dejean an de Gaulle, Mémoire, 21. 8. 1944, zitiert nach: Fritsch-Bournazel, Wende, S. 15.

2 S. Rede de Gaulles vor der Assemblée Consultative, 22. 11. 1944, in: de Gaulle, Discours, Bd. 1, S. 480-485; vgl. die kritischen Bemerkungen bei Hüser, „Doppelte Deutschlandpolitik“, S. 190194.

3 Zu Person und Politik de Gaulles vgl. vor allem Institut Charles de Gaulle, De Gaulle en son siècle, 7 Bde.; Lacouture, De Gaulle, 3 Bde.; s. a. Kap. V.1.1.

4 Vgl. Hüser, "Doppelte Deutschlandpolitik“, S. 91-111. 
sich General de Lattre de Tassigny, der Befehlshaber der 1. Französischen Armee, ausdrückte ${ }^{5}$, gleichzeitig die beste Gelegenheit, sich die als notwendig erachteten Faustpfänder zu sichern. Dabei ergab sich freilich „un tragique décalage entre l'image de la France et de la réalité française"6, stand doch der politische Anspruch in fundamentalem Widerspruch zur Wirklichkeit einer in ihren wirtschaftlichen und gesellschaftlichen Strukturen erschütterten, auf amerikanische Hilfe angewiesenen Mittelmacht”. Bisweilen mochte es gar wie ein „Unglück“ wirken, daß Frankreich zu den Siegern des Weltkrieges gezählt wurde. Denn das Bewußtsein, den Krieg gewonnen, aber die Schlachten verloren zu haben, führte zu einem „Komplex“, der die Nation daran hinderte, ihre globale Rolle „in den rechten Proportionen zu sehen“. Die Abhängigkeit von den USA schien wie ein „schweres Dilemma" auf ihr zu lasten. Akzeptierte sie die wirtschaftlich dringend notwendige Unterstützung, war sie in der Entwicklung eines eigenen außenpolitischen Programms gehemmt. Verweigerte sie sich der Hilfe, fehlte der französischen Weltpolitik die ökonomische Basis ${ }^{8}$.

De Gaulle versuchte die Zwangslagen und Widersprüche durch „Prestigebewußtsein und voluntaristische Unerbittlichkeit" zu überspielen". Mit Verve ging er sowohl hinsichtlich Deutschlands als auch in bezug auf die Beziehungen zu den Alliierten daran, seine Positionen für die Nachkriegszeit zu fixieren. Energisch betrieb er die Erlangung der völkerrechtlichen Anerkennung seiner Regierung, die aufgrund der amerikanischen Zögerlichkeit erst am 23. 10. 1944 erfolgte $^{10}$. Mit Beharrlichkeit forderte er die Aufnahme in die alliierten Entscheidungsgremien, insbesondere in die Commission Consultative Européenne (CCE), die Frankreich am 11. 11. erreichte ${ }^{11}$. Kurz vor dem Jahreswechsel konnte die Regierung außerdem den rückwirkenden Beitritt zur UNO zum 1. 1. 1942 verkünden ${ }^{12}$.

„Assurer d'une façon efficace et durable la sécurité de la France est la préoccupation essentielle du Gouvernement provisoire de la République française." So lautete die zentrale Maxime der französischen Außenpolitik. Und dies bedeutete neben der Wiederherstellung einer schlagkräftigen Armee vor allem die Beseitigung der deutschen Gefahr: „priver d'une façon définitive l'Allemagne des moyens matériels de faire la guerre, la placer et la maintenir dans une situation telle que toute nouvelle agression de sa part sera physiquement impossible ou vouée à un échec certain et rapide"13. Am nachhaltigsten schien dies mit den Mitteln der

5 De Lattre de Tassigny, Histoire, S. 472.

- Frank, Conclusions, S. 463; vgl. zum Problem der Machtperzeption in Frankreich ausführlich ders., Hantise.

7 Vgl. Bossuat, La France, Bd. 1, S. 25-98; Hüser, „Doppelte Deutschlandpolitik“, S. 43-90.

\& PA, Abt. 2, Bd. 236, Bl. 234-245, Aufzeichnung Frank, 3. 7. 1953; zur Bedeutung der amerikanischen Wirtschafts- und Finanzhilfen für die Formulierung der französischen Außenpolitik in den ersten Nachkriegsjahren vgl. grundsätzlich Bossuat, La France, 2 Bde.

9 Loth, Franzosen, S. 33; vgl. Linsel, Charles de Gaulle, S. 115 f.

10 S. Hoppenot an Bidault, Tel. 64, 28.9. 1944, in: DDF 1944, S. 42f.; Massigli an dens., Tel. 4876, 29. 9. 1944, Confidentiel, in: ebd., S. 43-45; Hoppenot an dens., Tel. 102, 16. 10. 1944, Secret, in: ebd., S. 127 f.; ders. an dens., Tel. 181, 19. 10. 1944, Réservé, in: ebd., S. 141 f.; Chauvel an London etc., Zirkulartel.178 etc., 24. 10. 1944, in: ebd., S. 155.

11 S. Massigli an Bidault, Tel. 375, 21. 11. 1944, in: ebd., S. 295 f.

12 S. Bidault an alle diplomatischen Vertretungen, Zirkulartel. 76, 29. 12. 1944, in: ebd., S. 508.

13 Aufzeichnung der französischen Delegation in Moskau, 1. 12. 1944, in: ebd., S. 343; vgl. Dejean an de Gaulle, Mémoire, 21. 8. 1944, in: Fritsch-Bournazel, Frankreich, S. 86. 
direkten und dauerhaften politischen und wirtschaftlichen Schwächung des daniederliegenden Nachbarn erreichbar. Seit September faßten die Verantwortlichen in Paris eine Besatzungszone ins Auge, die aus Teilen der englischen und amerikanischen gebildet werden sollte ${ }^{14}$. Am 27.12. beantragte Frankreich offiziell die Assoziierung ins Besatzungsregime für Deutschland, die Teilnahme an der Autorité suprême und für den Fall der deutschen Kapitulation die Übertragung einer Okkupationszone ${ }^{15}$. Wenige Wochen später gestanden ihm die Drei Großen auf der Konferenz von Jalta letzteres zu und gewährten die Aufnahme in den Alliierten Kontrollrat ${ }^{16}$.

Wie de Gaulle Anfang Dezember 1944 dem zum Directeur des affaires politiques im Quai d'Orsay aufgestiegenen Maurice Dejean auf der Reise nach Moskau und dann dem sowjetischen Diktator Stalin darlegte, sollten die linksrheinischen Gebiete unter ein besonderes, von Frankreich besetztes und verwaltetes Regime gestellt und die Ruhr internationalisiert werden, während er dem Rheinland insgesamt die Funktion eines "glacis" für Frankreich zuwies ${ }^{17}$. Natürlich hatte de Gaulle bei diesen konkreten Forderungen auch die öffentliche Meinung im Blick, doch handelte es sich gewiß um mehr als bloße "Ablenkungsangebote"18. Die jüngst veröffentlichten Dokumente aus dem Quai d'Orsay verdeutlichen eindrucksvoll die fundamentale Bedeutung, die der General gerade in der Allianz mit Moskau sah. Frankreich benötigte die Sowjetunion nicht nur zur Beseitigung der bestehenden und zukünftigen Gefahren aus dem Osten, sondern auch zur Durchsetzung seiner Interessen bei der Gestaltung der deutschen Nachkriegsordnung. Angesichts der gespannten Beziehungen zu den Westmächten suchte er gezielt die Nähe zur östlichen Flügelmacht und strebte zielbewußt auf einen möglichst engen Vertrag ${ }^{19}$. Gewiß verband de Gaulle mit dem Bündnis mit Stalin neben innenpolitischen Motiven ${ }^{20}$ auch die Absicht, die Anerkennung einer gleichberechtigten Großmachtposition von den Angelsachsen zu verlangen ${ }^{21}$. Doch der Pakt mit Moskau bedeutete aus seiner Sicht noch viel mehr; er war „le noeud“ jeglichen Sicherheitskonzepts gegen Deutschland22. Während die Sowjets an ein eher lockeres Bündnis dachten, zielte de Gaulle auf „une alliance pour une période indéfinie après cette guerre“. Einen von britischer Seite vorgeschlagenen dreiseitigen Pakt lehnte er aufgrund der bestehenden Meinungsverschiedenheiten im Nahen Osten

14 S. Massigli an Bidault, 30. 9. 1944, Très secret, in: DDF 1944, S. 47 f.; Aufzeichnung des Kabinetts von de Gaulle, 23. 10. 1944, in: ebd., S. $151 \mathrm{f}$.

15 S. Aufzeichnung der Französischen Delegation bei der CCE, 27. 12. 1944, Secret, in: ebd., S. 489491; Bidault an Massigli, Nr. 719, 29. 12. 1944, Secret, in: ebd., S. 510f.; ders. an Washington u. Moskau, Tel. 533 u. 192, 30. 12. 1944, Réservé, in: ebd., S. $511 \mathrm{f}$.

16 Zum Konferenzverlauf vom 4.-11. 2. 1945 vgl. Graml, Alliierte, S. 31-60; zur Geschichte des Alliierten Kontrollrats vgl. die profunde Studie von Mai, Kontrollrat.

17 Aufzeichnung der französischen Delegation in Moskau, 1. 12. 1944, in: DDF 1944, S. 343-345, hier S. 345; s. a. Unterredung zwischen de Gaulle und Stalin vom 8. 12. 1944, Très secret, in: ebd., S. $419-422$.

18 Hüser, „Doppelte Deutschlandpolitik“, S. 256.

19 S. den französisch-sowjetischen Bündnisvertrag, 10.12. 1944 in: EA 1947, S. 1046; vgl. Narinsky, De Gaulle.

20 Vgl. Hüser, „Doppelte Deutschlandpolitik“, S. 105.

21 S. DePorte, Foreign Policy, S. 79; Linsel, Charles de Gaulle, S. 119-121.

22 Aufzeichnung der französischen Delegation in Moskau, 1. 12. 1944, in: DDF 1944, S. 343. 
und der erwarteten Differenzen in der Deutschlandpolitik ab ${ }^{23}$. Allenfalls unterhalb der Ebene der bilateralen Allianz mit der Sowjetunion konnte er sich ein trilaterales Bündnis mit britischer Beteiligung vorstellen, und darunter wiederum ein kollektives Sicherheitssystem unter Einschluß der Amerikaner. „Du point de vue de la France", betonte der französische General gegenüber seinem sowjetischen Gastgeber, „'alliance franco-soviétique vient donc en premier lieu“, damit man präventiv agieren und sofort jeder äußeren Aggression begegnen könne24.

Um den sperrigen Wunschpartner auf seine Seite zu ziehen, lockte de Gaulle insbesondere in der polnischen Frage mit Konzilianz und akzeptierte ausdrücklich die Oder-Neiße-Grenze. Darüber hinaus kam er dem Generalissimus auch noch in anderer Hinsicht entgegen. Entgegen den seit 1943 angedachten Planungen einer westeuropäischen Föderation unter Einschluß von Ruhr und Rheinland ${ }^{25}$ ließ er jetzt seine Bereitschaft erkennen, die Ruhr ebenso wie das Rheinland vom Reichsgebiet abzutrennen, ohne es einem gemeinsamen Regime zu unterstellen. Während das Rheinland französisch überwacht werden sollte, sah er für die Ruhr die Internationalisierung vor. Doch Stalin verweigerte die Unterstützung ${ }^{26}$. Das Grundkonzept der „géopolitique à la française“, Kontrolle Deutschlands mit sowjetischer Hilfe und Kontrolle der Sowjetunion mit einem von Frankreich geführten geeinten Westeuropa, blieb aber prinzipiell erhalten ${ }^{27}$.

Im Quai d'Orsay stießen die Planungen des Chefs im Matignon keineswegs auf ungeteilte Zustimmung, ohne daß man ihnen zu diesem Zeitpunkt bereits konkrete eigene Vorstellungen entgegengesetzt hätte. Als die Beamten in einer Arbeitssitzung im November Pläne zur Bildung eines „État rhéno-westphalien“ diskutierten, favorisierten sie die Vereinigung des westfälisch-rheinischen Territoriums mit der Mosel- und Lahnregion, eventuell auch noch mit dem Saar- und Nahegebiet. Zugleich sprachen sie sich gegen eine gemeinsame Grenze des neuen Staates mit dem Elsaß und für eine lange Besatzungszeit aus. Mit Blick auf die Saar plädierten sie für die Unterstellung unter ein Mandat „au bénéfice de la France avec transferts de population“28. "Parmi les différents régimes à envisager", so heißt es in einer Aufzeichnung des Quai d'Orsay Anfang Dezember zur Saar, „un seul satisfait toutes les exigences de l'économie française: c'est l'annexion politique." Neben den strategischen Vorteilen garantiere sie vor allem die erhofften Vorteile der wirtschaftlichen Annexion. Allerdings stand ihr der Wille einer homogenen Bevölkerung entgegen, und sie zu teilen sei aussichtslos, ein Bevölkerungsaustausch international kaum durchzusetzen. Als Alternative hielt das

23 Unterredung zwischen de Gaulle und Bogomolov vom 7. 12. 1944, Très secret, in: ebd., S. 410f., hier S. 410.

24 Unterredung zwischen dems. und Stalin vom 8. 12. 1944, Très secret, in: ebd., S. 419-422, hier S. 420.

25 Vgl. Poidevin, Französische Deutschlandpolitik; Soutou, Place, S. 45; Wilkens, Neuordnung.

26 S. Unterredung zwischen de Gaulle und Stalin vom 6. 12. 1944, Très secret, in: DDF 1944, S. 386394; Unterredung zwischen dens. vom 8. 12. 1944, Très secret, in: ebd., S. 419-422.

27 Soutou, Place, S. 46.

28 Unterredung im MAE unter der Leitung von Alphand vom 9. 11. 1944, Protokoll, in: DDF 1944, S. 232-235, hier S. 235; s. a. Unterredung im MAE unter der Leitung von Chauvel vom 27.12. 1944, Protokoll, in: ebd., S. 491-494, Soutou, Anschluß des Saarlandes?, S. 227. 
Ministerium ein internationales Statut für die beste Lösung, sofern es definitiv gelte 29 .

Ungeachtet der Differenzen in Regierung und Verwaltung 30 ließ de Gaulle mit seinen Reden, Erklärungen und diplomatischen Initiativen nach dem Jahreswechsel keinen Zweifel an der Aufrechterhaltung seiner deutschlandpolitischen Forderungen: Angliederung des Saargebietes an Frankreich, dauerhafte Abtrennung des Rheinlandes vom übrigen Deutschland, Anerkennung der Oder-Neiße-Grenze, Unterwerfung des Ruhrgebietes unter internationale Kontrolle, massive Demontagen und Reparationsleistungen ${ }^{31}$.

Daß die extensive Nutzung der deutschen Ressourcen nur kurzfristig andauern und dann der sukzessiven Integration einer kontrollierten deutschen Wirtschaft in den europäischen Rekonstruktionsprozeß folgen sollte ${ }^{32}$, scheint kaum glaubwürdig eingedenk der eindeutigen Pläne Frankreichs, die Modernisierung der eigenen Wirtschaft mit Hilfe der Zerstörung der deutschen Ökonomie aufzubauen ${ }^{33}$. Allen Verantwortlichen in Paris war klar, daß das Sicherheitsproblem künftig weitgehend wirtschaftlicher Natur sein würde. Für das militärische Niederhalten Deutschlands sorgten die alliierte Solidarität und der Pakt mit der Sowjetunion, im März 1947 noch ergänzt durch die Allianz mit Großbritannien ${ }^{34}$. Wie aber konnte man sich der ökonomischen Potenz des östlichen Nachbarn auf Dauer erwehren? Indem Deutschland als Machtfaktor ausgeschaltet und Frankreich nicht zuletzt mit Hilfe des ökonomischen Potentials des Nachbarn als Vormacht auf dem europäischen Festland etabliert würde. Der Abbau der deutschen Wirtschaftskraft sollte dazu genutzt werden, aus Frankreich „eine industrielle Großmacht zu machen" ${ }^{35}$. Mit amerikanischen Krediten und deutschen Ressourcen gedachten die Verantwortlichen in Paris der geschundenen "Grande Nation" den überfälligen Schritt zur wirtschaftlichen und gesellschaftlichen Modernisierung zu erleichtern ${ }^{36}$. Mit der Abtrennung des Ruhrgebietes und der dortigen Installierung einer von den Siegermächten abhängigen Regierung ${ }^{37}$ wollten sie einerseits die "Waffenschmiede" des deutschen Militarismus beherrschen. Andererseits war die Sicherung einer ausreichenden Kohleversorgung das zentrale Element, mit dem der von Jean Monnet und seinem Stab ausgearbeitete Modernisierungsplan stand und fiel ${ }^{38}$.

Unter dem Motto „Plus de Reich centralisé!“ verlangte der General darüber hinaus eine Aufteilung des „corps germanique“ in mehr oder minder locker ver-

29 Aufzeichnung der Abt. für wirtschaftliche Angelegenheiten im MAE, 4. 12. 1944, in: ebd., S. 357368, hier S. 365; s. a. Soutou, Anschluß des Saarlandes?, S. 227 f.

30 S. Burin des Roziers an Palewski, Aufzeichnung, 30. 12. 1944, in: DDF 1944, S. $514 \mathrm{f}$.

31 Mit zahlreichen Belegen vgl. Lipgens, Bedingungen; Poidevin, Französische Deutschlandpolitik, S. 15-22; ders., Frankreich und die Deutsche Frage, S. 408-414; Soutou, Anschluß des Saarlandes?, S. 228-235; s. a. die Dokumentation in: EA 1946/47, S. 266-272.

32 Vgl. Hüser, „Doppelte Deutschlandpolitik“, S. 398.

33 Vgl. Bossuat, La France, Bd. 1, S. 63-81 u. 293 f.; Lefèvre, Relations, S. 20-32.

34 Vgl. Bell, France, S. 68-84.

35 Aufzeichnung des Directeur général des Affaires économiques, financières et techniques im MAE, Alphand, 24. 7. 1945, zitiert nach: Fritsch-Bournazel, Wende, S. 10.

36 Vgl. Loth, Deutsche Frage, S. 39.

37 Zur Bedeutung der Ruhrfrage für Frankreich 1945-1949 vgl. Gillingham, Coal, S. 148-173; Poidevin, Frankreich und die Ruhrfrage; ders., La France et le charbon allemand.

38 Vgl. Mioche, Plan Monnet. 
bundene Einzelstaaten ${ }^{39}$. „Rücksichtslos“, so mochte es aus der deutschen Sicht scheinen, nutzte Frankreich seine Stellung als Besatzungsmacht aus und betrieb nach Abschluß der Potsdamer Konferenz vom August 1945, zu der es bekanntermaßen nicht eingeladen worden war, eine „Politik der Spaltung Deutschlands“" indem es im Alliierten Kontrollrat die vereinbarten deutschen Zentralverwaltungen obstruierte ${ }^{41}$. An der Zerstörung der deutschen Wirtschaftseinheit war man dagegen - mit Ausnahme der Saar - nicht interessiert und entwickelte daher im Herbst das Konzept der „bureaux alliés“, deutsche Verwaltungsstellen unter Aufsicht des Kontrollrates ${ }^{42}$.

Die von der Regierungsspitze an den traditionellen Mustern ausgerichtete „harte“ Deutschlandpolitik entsprach dem Willen einer großen Mehrheit der französischen Bevölkerung, in der Mißtrauen, $\mathrm{Haß}$ und Rachegefühle gegenüber dem östlichen Nachbarn überwogen und $\mathrm{zu}$ "gravierenden Minderwertigkeitskomplexen" führten"3. Freilich darf von der Germanophobie weiter Bevölkerungskreise ebensowenig auf eine ausschließliche Unterstützung des Dominanzkonzeptes geschlossen werden ${ }^{44}$ wie von der ebenfalls feststellbaren breiten $\mathrm{Zu}$ stimmung zur europäischen Integration ${ }^{45}$ auf eine einheitliche Absage dieses Konzeptes ${ }^{46}$. Gleichwohl gab es durchaus alternative deutschlandpolitische Ideen, wie sie in Stellungnahmen von Parteien, Schriftstellern, Publizisten oder Kirchenvertretern zum Ausdruck kamen ${ }^{47}$. Freilich gab es in der intellektuellen Elite auch Stimmen, die für eine langfristige Niederhaltung des besetzten Deutschland plädierten, wie François Mauriac und Edmond Vermeil ${ }^{48}$. Demgegenüber diskutierten insbesondere Vertreter der sozialistisch inspirierten Résistance Pläne, die vom Ausgleich mit Deutschland im Rahmen einer neuen europäischen Staatenordnung bestimmt waren und vor allem in der SFIO auf fruchtbaren Boden fielen ${ }^{49}$. Persönlichkeiten verschiedener politischer Couleur warnten die Regierung eindringlich vor der Versuchung der Revanche und redeten einer Politik der Verständigung das Wort - so etwa Henri Frenay, der Gründer der Résistance-Gruppe "Combat“, oder Raymond Aron, der in London das Sprachrohr „La France libre“

39 De Gaulle, Mémoires de Guerre, Bd. 3, S. 46. Für Hüser zielt diese Formulierung nicht auf eine Zerteilung, sondern lediglich auf eine möglichst weitreichende Dezentralisierung (Hüser, „Doppelte Deutschlandpolitik“, S. 260f.). Hudemann hält beide Interpretationen für legitim (Hudemann, Reparationsgut, S. 38).

$40 \mathrm{PA}, \mathrm{Abt}$. 2, Bd. 249, Bl. 315-327, „Enttäuschungen in unseren Bemühungen um ein gutes Verhältnis mit Frankreich“, o.D. Die Aufzeichnung wurde Staatssekretär Hallstein am 17.11. 1953 zugestelit (ebd., Bl. 314, Strohm an Hallstein, 210-01/22 III 24726/53, 17.11. 1953).

41 Vgl. Bungert, New Perspective; Deuerlein, Obstruktion; Kraus, Ministerien; Mai, Kontrollrat, S. 83-92.

42 Vgl. Hudemann, Weichenstellungen, S. 136 f.; ders., Frankreich und der Kontrollrat. Henke hingegen verweist darauf, daß es Frankreich bei einer zentralen Wirtschaftsverwaltung Deutschlands "nicht möglich gewesen [wäre], die ansehnlichen Wirtschaftsgewinne, die es in seiner Zone zu erzielen verstand, nach Frankreich zu transferieren, wo sie fest in die Planung der ökonomischen Rekonstruktion integriert waren" (Henke, Aspekte, S. 70); zum Thema Wirtschaftseinheit vgl. ausführlich Mai, Kontrollrat, S. 173-255.

43 Hüser, „Doppelte Deutschlandpolitik“, S. 64.

44 Vgl. dagegen Hänsch, Frankreich.

45 Vgl. Loth, Franzosen, S. 32.

46 Vgl. demgegenüber: Lipgens, Kritik, S. 164-198.

${ }^{47}$ Vgl. ausführlich Lipgens, Kritik.

48 Vgl. Mortier, Mythe, S. 38-55; Auerbach, „Que faire?“.

49 Vgl. die grundlegende Arbeit von Loth, Sozialismus. 
redigiert hatte ${ }^{50}$. Einen kaum zu überschätzenden Beitrag zum produktiven Miteinander lieferten privat ausgerichtete Gesellschaften dies- und jenseits des Rheins ${ }^{51}$. Auf Initiative des Jesuitenpaters Jean du Rivau riefen das „Bureau International de Liaison et de Documentation“ und die „Gesellschaft für übernationale Zusammenarbeit“ 1945 das dem Dialog verpflichtete Zeitschriftenpaar „Documents" bzw. „Dokumente" ins Leben ${ }^{52} .1948$ gründeten Emmanuel Mounier und Alfred Grosser die Einrichtung des „Comité d'échanges avec l'Allemagne nou-

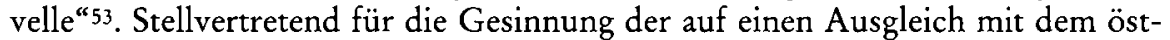
lichen Nachbarn bedachten Kreise mag der häufig zitierte Appell dienen, den Joseph Rovan 1945 in der Zeitschrift „Esprit“ veröffentlichte: „L'Allemagne de nos mérites" 54 .

Auch in den verschiedenen Ministerien ${ }^{55}$, sogar im französischen Generalstab gab es Stimmen, die energisch davon abrieten, die Politik der Zwischenkriegszeit zu wiederholen. Aus dem „Pyrrhussieg“56 von 1918 zogen sie die Lehre, dem stets unruhigen Nachbarn Wege zu öffnen, „selon lesquelles il pourra s'épanouir, et

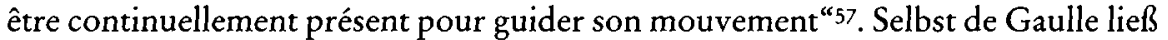
während einer Reise durch die französische Besatzungszone im Herbst 1945 konziliantere Töne erklingen. Er reagierte damit auf die Widerstände, auf die seine Politik bei den westlichen Alliierten stieß, und auf die wachsende Unruhe, die ihm die sowjetische Politik einflößte ${ }^{58}$. Seine Reden und Ansprachen ließen aber noch etwas anderes erkennen. Auch die Verfechter eines karthagischen Friedens waren sich der Tatsache bewußt, daß die Zukunft Deutschlands im Mittelpunkt des europäischen Problems stehen mußte ${ }^{59}$.

Der Rücktritt de Gaulles am 20. 1. 1946 bedeutete „keine Zäsur für die französische Deutschlandpolitik"60. Die Einbeziehung von Kommunisten in die vom Sozialisten Félix Gouin fortgesetzte Koalition des Tripartisme und die antideutsche Haltung der Öffentlichkeit verboten eine Kehrtwende ${ }^{61}$. Die ausgesprochen instabile Situation des politischen Nachkriegsfrankreich behinderte ohne Zweifel die Formulierung einer kontinuierlichen Deutschlandpolitik. Zwischen den ersten Wahlen zur Nationalversammlung im November 1946 und dem zweiten Urnengang im Juni 1951 lösten sich neun Kabinette in den Regierungsgeschäften ab. Auch wenn nur drei Außenminister in diesem Jahrfünt amtierten, hatten diese doch die unterschiedlichen Koalitionen in ihrer Politik zu berücksichtigen. Auch

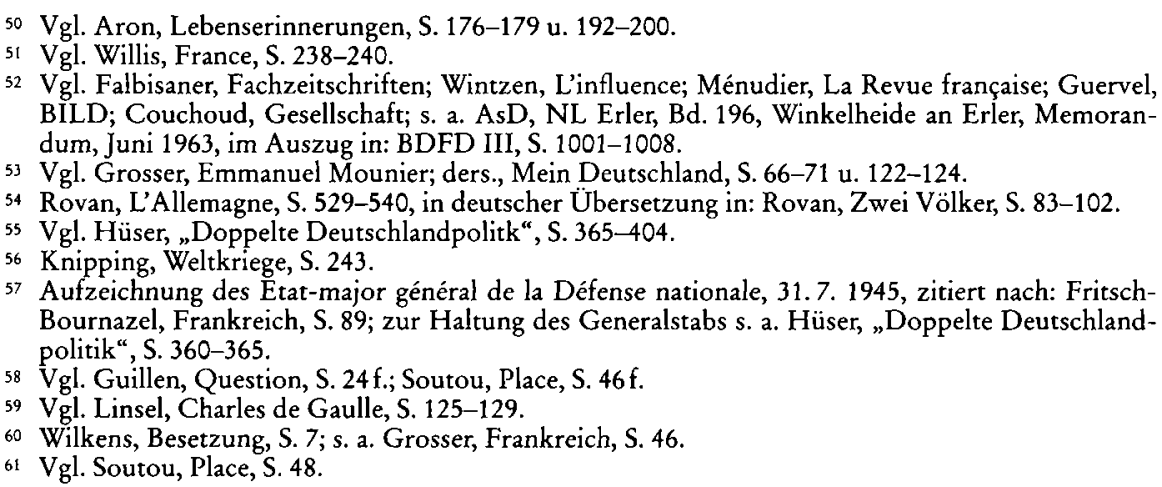


mußten sie mit einer Nationalversammlung rechnen, die in außen- und deutschlandpolitischen Angelegenheiten mitzureden suchte und in der die Fronten meist durch die Parteien gingen ${ }^{62}$. Allerdings setzte das Kabinett Gouin vorsichtig neue Akzente. Die "déboires sur le plan territorial" ließen es um so energischer den wirtschaftlichen Aspekt der Sicherheitsfrage in den Vordergrund stellen: Schwächung des ökonomischen Potentials und dauernde Kontrolle der wichtigsten Industrieregionen ${ }^{63}$. So betonte Frankreich die Notwendigkeit einer anhaltenden militärischen Besetzung Deutschlands und forderte weiterhin eine umfassende internationale Lösung für die Ruhr, nicht aber die Abtrennung von Rheinland und Ruhrgebiet ${ }^{64}$.

Wichtiger als Gouin war für den Verlauf der französischen Deutschlandpolitik der bereits unter de Gaulle amtierende Außenminister Georges Bidault. Jahrgang 1899, stammte er aus dem unweit Vichy gelegenen Städtchen Moulins. Er studierte Geschichte bzw. Geographie und arbeitete dann als Gymnasiallehrer. Nach dem Beitritt und dem raschen Aufstieg in das Exekutivkomitee des Parti Démocrate Populaire trat er 1934 in die Redaktion von „l'Aube“ ein, der Zeitschrift der christdemokratischen Bewegung. Im Anschluß an die deutsche Kriegsgefangenschaft begann 1941 seine eigentliche politische Laufbahn. Bidault schloß sich dem Widerstand an und übernahm 1943 von Jean Moulin den Vorsitz des Conseil National de la Résistance. Im August 1944 leitete er den Pariser Aufstand gegen die Deutschen, der am 26.8. mit dem Triumphzug über die Champs Elysées endete, für Bidault aber gleichzeitig die erste Auseinandersetzung mit de Gaulle brachte. Seiner Aufforderung, die Republik auszurufen, verweigerte sich der General, weil diese seiner Meinung nach nie untergegangen war. Außerdem gefiel es ihm nicht, von Bidault Befehle entgegennehmen zu sollen. Auf dem Marsch über die Pariser Prachtstraße zog de Gaulle ihn von der rechten auf seine linke Seite und ließ ihn einige Meter hinter sich. Die Hierarchie war für alle sichtbar dokumentiert. Für die weitere Zusammenarbeit bildete sie kein Hindernis. Im September zog Bidault in den Quai d'Orsay ein, den er bis 1954 insgesamt dreimal leitete. 1946 und 1949/ 50 amtierte der Mitbegründer des christdemokratischen „Mouvement Républicain Populaire" (MRP) als Regierungschef, 1951/52 leitete er das Verteidigungsressort. Zwei Jahre später führte seine politische Karriere zunächst in die „marginalisation " 65 und endete schließlich in der Isolation. Bidaults entschiedene Gegnerschaft gegen die von de Gaulle nach seiner Rückkehr zur Macht 1958 in Angriff genommene Dekolonisationspolitik brachte ihn in die Nähe zur extremen Rechten. Als Mitglied des Exekutivkomitees der „Organisation de l'Armée Secrète" zwang ihn der mißglückte OAS-Putsch 1962 ins Exil, das er bis zur Amnestie 1968 in Brasilien verbrachte ${ }^{66}$.

Brillant, aber schroff, mit festgefügtem Weltbild, aber auch „machiavellistischem Naturell" 67 versehen, als begabter Redner eher zu bissigen Bemerkungen

$62 \mathrm{Vgl}$. Kiersch, Parlament, bes. S. 187-223.

63 Guillen, Question, S. 25.

64 Vgl. Rede Gouins, 24. 3. 1946, in: L'Année politique 1946, S. 532 f.; Hitchcock, France Restored, S. $59 f$.

65 Dalloz, Georges Bidault, S. 384.

66 Zu Person und Politik Bidaults vgl. ebd.; Demory, Georges Bidault; Vaïse, Georges Bidault.

67 PA, Abt. 3, AZ 215-03/22, Bd. 1, Hausenstein an AA, 731-01 Tgb. Nr. 4140/52, 29. 10. 1952. 
denn ausgefeilten Analysen neigend, haftete dem im katholischen Glauben verankerten Christdemokraten mit Idealen und Ideen das Stigma der Unberechenbarkeit an ${ }^{68}$. Dem Deutschlandproblem setzte er mit augenscheinlicher „soviétophilie $^{\text {"69 }}$ zunächst den Versuch einer engen Bindung zur Sowjetunion in Anlehnung an dem von ihm unterzeichneten Pakt von 1944 und die Fortsetzung der von de Gaulle vertretenen harten Linie entgegen. Sie entsprach dem weiterhin vorherrschenden negativen Meinungsklima gegenüber Deutschland ${ }^{70}$ sowie dem eigenen Machterhalt. Denn gerade die Christdemokraten konnten es nicht wagen, von dem bisherigen Kurs abzuweichen, wollten sie nicht große Teile ihrer Anhänger an "gaullistische" Formationen verlieren"1. Mochte er auch allmählich an den Erfolgsaussichten der Deutschlandpolitik zweifeln ${ }^{72}$, hielt er ungeachtet der zunehmend deutlicher werdenden fehlenden Zustimmung der Westmächte seine Positionen aufrecht, um die innenpolitische Machtbasis nicht zu verlieren. Denn die Forderung nach Rheinland-Separierung oder Ruhr-Internationalisierung hatte für viele Franzosen „identitätsstiftenden Symbolcharakter"73 gewonnen. Auch glaubte er, so besser wirtschaftliche Ansprüche bei den Westmächten durchzusetzen $^{74}$. Insgeheim aber konzipierte er im Bewußtsein der fehlenden Durchsetzbarkeit maximalistischer Forderungen und vor dem Hintergrund der wachsenden Furcht vor der Sowjetunion und einer „conjonction soviéto-allemande" ${ }^{\text {" } 75}$ mit seinen Mitarbeitern im Quai d'Orsay wie Chauvel, dem Generalsekretär, Massigli, dem Botschafter in London oder Bonnet, dem Vertreter in Washington, die Wende $^{76}$. Daß die alte Deutschlandpolitik offiziell keineswegs aufgegeben wurde, verdeutlichte der aus Sorge vor einem deutsch-sowjetischen Bündnis geschlossene französisch-britische Vertrag von Dünkirchen vom 4.3. 1947, der ausdrücklich gegen Deutschland gerichtet war, „et contre elle seule“, zugleich aber dem Wunsch französischer und britischer Sozialisten nach einer Dritten Kraft als Mittler zwischen den Weltmächten entsprach77.

Mehr und mehr wurden sich die französischen Politiker der Tatsache bewußt, daß die Durchsetzung der offiziellen Politik kaum mehr als eine Fiktion war, wie vor allem die Gründung der Bizone am 1. 1. 1947 unter Beweis stellte. Mit der europapolitische Verantwortung übernehmenden Verkündung der Truman-Doktrin am 12. 3. und dem Scheitern der Moskauer Außenministerkonferenz (10.3. bis 25. 4. 1947) setzte dann die entscheidende Etappe bei der Neuorientierung der französischen Deutschlandpolitik ein. Bidaults Hoffnung, zwischen den angel-

68 Vgl. Grosser, Frankreich, S. 105; Vaïsse, Georges Bidault, S. $81 \mathrm{f}$.

69 Couve de Murville, zitiert nach: Dalloz, Georges Bidault, S. 105.

70 Vgl. Hänsch, Frankreich, S. 18-39.

71 Zur Außen- und Deutschlandpolitik des MRP vgl. Capelle, MRP; Irving, Christian Democracy, S. 159-198; Schreiner, Bidault.

72 Ob Bidault freilich bereits seit der Jahreswende 1945/46 nicht mehr an die Zweckmäßigkeit der „harten“ Deutschlandpolitik glaubte (Hüser, „Doppelte Deutschlandpolitik“, S. 266-282), muß erst noch durch weitere Forschungen bestätigt werden.

73 Loth, Deutsche Frage, S. 41.

74 Vgl. ebd., S. 42; Schreiner, Bidault, S. 117; am weitesten in diesem Sinne: Gillingham, Ruhrpolitik, S. 9.

75 Santiard, Diplomates français, S. 155.

$76 \mathrm{Vgl}$. Soutou, Place, S. $48 \mathrm{f}$.

77 Ebd., S. 49. 
sächsischen und sowjetischen Gegensätzen eine eigenständige Position verteidigen zu können, zerschlug sich endgültig. Der sowjetische Außenminister Molotow widersetzte sich energisch allen französischen Sonderplänen und forderte die Errichtung zentraler deutscher Verwaltungsstellen, die von Bidault abgelehnt wurden. Zugleich mißbilligte Molotow die wirtschaftliche Bindung des Saarlandes an Frankreich, während Engländer und Amerikaner ihr Einverständnis mit der Wirtschaftsfusion signalisierten und einer Erhöhung des Kohleexportes aus den Westzonen nach Frankreich zustimmten ${ }^{78}$. Bidault verschrieb sich nun endgültig dem westlichen Lager: „l'entente avec l'URSS est impossible, il a tenté jusque-là loyalement de se rapprocher de Moscou, mais il est désormais décidé à jouer la carte américaine“79. Offenbar kalkulierte der Quai d'Orsay den Mißerfolg indes bereits ein, begrüßte ihn in gewisser Weise sogar, um das schrittweise Einschwenken auf die angelsächsische Linie nach innen begründen zu können ${ }^{80}$. Daß die sich formierende Bildung der Blöcke den eigenen Handlungsspielraum „entscheidend zusammenschrumpfen " ließ, konnte einer nach Weltgeltung strebenden französischen Führung natürlich nicht gefallen ${ }^{81}$. Fast resignativ gestand Bidault sich nun ein, daß die Politik der "Grandeur" gescheitert war ${ }^{82}$.

Ohne abrupten Umschlag von einer harten zu einer kooperativen Deutschlandpolitik gewann nach der Konferenz diejenige Tendenz die Oberhand, die die Weichen auf eine langfristige kooperative Kontrolle des deutschen Nachbarn stellen wollte ${ }^{83}$. Wenn dieses Einschwenken auf die anglo-amerikanische Linie „mehr oder minder von außen auferlegt worden war"84, dann doch wohl nur in dem Sinne, daß Frankreich aus einer veränderten außenpolitischen Konstellation selbständig die entsprechenden Einsichten entwickelte. Verschiedene Faktoren zeichneten dafür verantwortlich: Auf der internationalen Ebene trat mit der Verschärfung des Ost-West-Konfliktes - wie sie insbesondere die kommunistische Machtergreifung in der Tschechoslowakei unter Beweis stellte - das Bewußtsein von der Gefahr einer sowjetischen Expansion in den Vordergrund. Die Verfechter der Unabhängigkeit Frankreichs sahen sich zur Intensivierung ihres Projektes eines Europa der „Dritten $\mathrm{Kraft}^{\text {“ }}$ zwischen den Weltmächten gedrängt und leiteten unter Léon Blum das Bündnis mit Großbritannien als Kernstück dieser Konzeption ein, ohne zuvor, wie de Gaulle und Bidault es stets verlangt hatten, Zugeständnisse hinsichtlich der eigenen Abtrennungsforderungen zu verlangen ${ }^{85}$.

Erleichtert wurde die Umorientierung durch den innenpolitischen Wechsel nach der Entlassung der kommunistischen Minister aus der Regierung Ramadier im Mai 1947, die die Kriegsallianz auch in der Regierungskoalition zerfallen ließ und den Einfluß der Sozialisten spürbar vergrößerte ${ }^{86}$. Die innenpolitischen Fron-

78 Vgl. Kessel, Westeuropa, S. 211-256; Kerkhoff, Großbritannien, S. 55-61; Pohlmann, Saarfrage, S. 154-186; zur Bedeutung der Truman-Doktrin vgl. Soutou, Place, S. 49.

79 Dalloz, Georges Bidault, S. 171.

80 Vgl. Auerbach, Wende; Kessel, Westeuropa, S. 160-177; Young, France, S. 134-154.

81 Wilkens, Besetzung, S. 10.

82 Hitchcock, France Restored, S. 72.

83 Vgl. Bungert, New Perspective, S. 352.

84 Fritsch-Bournazel, Frankreich, S. 93.

85 Vgl. Loth, Franzosen, S. 39 f.

86 Vgl. Fonvieille-Vojtovic, Paul Ramadier, S. 321-358; Loth, Sozialismus, S. 139-143. 
ten klärten sich damit. Die Vertreter einer harten Hand - Gaullisten und Kommunisten - saßen in der Opposition, während Bidault nun stärker der kooperationswilligen Linie der SFIO Rechnung zu tragen hatte ${ }^{87}$. Darüber hinaus war bei den Entscheidungsträgern angesichts der schwierigen Versorgungslage, der finanziellen und militärischen Schwäche das Bewußtsein der Abhängigkeit von der amerikanischen Marshallplanhilfe gewachsen, die zu erhalten bei einer Fortsetzung der bisherigen Linie nicht den Hauch einer Chance besaß88. Im Zeichen von Lebensmittelverknappung und schwindenden Devisenreserven wirtschaftlich am Ende ${ }^{89}$, sich des Dilemmas „modernisation sous influence ou l'indépendance dans la décadence " 90 voll bewußt, entschied sich Paris im Herbst für ein Einschwenken auf den anglo-amerikanischen Kurs. Paradoxerweise wurden die wirtschaftlichen Bedürfnisse Frankreichs, die wesentlichen Anteil an der deutsch-französischen Spannung hatten, ab 1947 zu einem Element der Annäherung. Damit geriet die französische Politik zwar nicht zum "Wurmfortsatz" der amerikanischen Deutschlandpolitik ${ }^{91}$, aber die Handlungsmarge fand sich angesichts des angelsächsischen „Harmonisierungsdruck $[\mathrm{s}]^{\text {“92 }}$ ohne Zweifel eingeengt.

Nach dem Scheitern der Londoner Tagung des Außenministerrates vom 25. 11. bis $15.12 .{ }^{93}$, das den Bruch der alliierten Koalition besiegelte, wurde der Wandel der Deutschlandpolitik deutlicher konturiert. Im Selbstverständnis des Quai d'Orsay begann in seiner Besatzungspolitik nun ein neuer Zeitabschnitt, der nicht weniger bedeutete, als daß eine „konstruktive“ einer „nur negativen Politik“ Platz zu machen und er nunmehr von der Prämisse auszugehen hatte, „daß wir in eine neue Periode eintreten, in der das vorherrschende Verlangen nicht mehr der Bezug auf die Vergangenheit und [...] ihre Wiedergutmachung sein soll, sondern die Vorbereitung der Zukunft"94. Mit der Londoner Sechsmächtekonferenz setzte im Frühjahr bzw. Frühsommer 1948 die letzte Phase der französischen Umorientierung ein. Das „besatzungsrechtliche Einzelgängertum “ 95 wurde nun schrittweise aufgegeben. Zwar konnte die französische Delegation sich nicht mit der Forderung nach einer internationalen Hoheit über die Ruhr durchsetzen, erzielte aber den Kompromiß der Kontrolle über die Verteilung von Kohle und Stahl - nicht aber der Produktion - sowie ein Aufsichts- und Kontrollrecht über die Aktivitäten der Bergwerke und Stahlfabriken. Im Gegenzug mußte sie endgültig dem amerikanisch-britischen Verlangen nach Bildung eines westdeutschen Staates nachgeben ${ }^{96}$.

Genugtuung oder Zufriedenheit über die sich abzeichnende Zweiteilung Deutschlands läßt sich in den Meinungsäußerungen der französischen Diplomatie

87 Vgl. ebd., S. 167-169.

88 S. Erklärung Bidaults im Conseil des Ministres vom 26. 5. 1948, in: Auriol, Journal, Bd. 2, S. 241.

89 Zur wirtschaftlichen Situation Frankreichs 1947 vgl. Bossuat, La France, Bd. 1, bes. S. 99-139.

90 Frank, Dilemme français, S. 137.

91 Kiersch, Deutschlandpolitik, S. 76; ähnliche Urteile bei Korff, Revirement, S. 74-87; Ziebura, Beziehungen, S. 62-68.

92 Knipping, "Que faire?", S. 147.

93 Zum Konferenzverlauf vgl. Kessel, Westeuropa, S. 282-294; Mai, Kontrollrat, S. 449-464.

94 Instruktionen des MAE Nr. 51/EU, 15. 1. 1948, zitiert nach: Wilkens, Besetzung, S. 13; s. a. Hitchcock, France Restored, S. 89-93.

95 Kolboom, Frankreich, S. 73.

$96 \mathrm{Zu}$ dieser Konferenz jetzt grundlegend: Wehner, Westalliierte. 
kaum nachweisen. Die Stimme Tarbé de Saint-Hardouins, des Politischen Beraters der französischen Militärregierung in Berlin, vom Oktober 1948, die Spaltung der Kriegsalliierten habe wenigstens die Konsequenz, „uns als Nachbarn einen Staat mit 45 Millionen Einwohnern anstatt mit 70 Millionen" zu geben" 9 , bleibt die Ausnahme. Allerdings stießen die Londoner Beschlüsse in der französischen Öffentlichkeit wie im Kabinett auf herbe Kritik. Couve de Murville, Generaldirektor für politische Angelegenheiten im Quai d'Orsay und ein „Hardliner in deutschen Angelegenheiten "98, weigerte sich, die Übereinkunft im Auswärtigen Ausschuß der Nationalversammlung zu vertreten ${ }^{99}$. Doch Bidault hielt seinen Kollegen entgegen: „Ce que nous ne ferons pas avec les autres, ils le feront sans nous et nous serons asphyxiés, car nous n'aurons rien. " 100 Nur mit der knappen Mehrheit von 300 zu 286 Stimmen stimmte die Assemblée Nationale den Londoner Entscheidungen am 16. 6. zu'101. Damit war der entscheidende Eintritt Frankreichs in die westliche Allianz vollzogen. Die Wahl wurde aber keineswegs als irreversibel betrachtet, wie die großen Pressepolemiken anläßlich der Gründung der Atlantischen Allianz im Mai 1949 unter Beweis stellen sollten; mit ihrer erstmaligen eindeutigen Wendung gegen Moskau bedeutete der Vorgang für viele Franzosen geradezu eine "révolution psychologique“ ${ }^{102}$.

Nachdem sich Frankreich mit der Grundsatzentscheidung über die Schaffung des neuen deutschen Staates abgefunden hatte, zielte es nun darauf, die innere Ausgestaltung nach seinen Vorstellungen mitzubestimmen. Besondere Bedeutung kam dabei der Verfassung zu, für die man im Vergleich zu den Angelsachsen die konkretesten Pläne ausarbeitete ${ }^{103}$. Die dezidiert antizentralistischen Entwürfe, wie sie Bidault bereits im Januar 1947 vorgelegt hatte ${ }^{104}$, ließen sich ebensowenig realisieren wie das noch auf der Londoner Sechs-Mächte-Konferenz verfolgte Ziel eines Staatenbundes ${ }^{105}$. Entsprechend seiner in der eigenen Besatzungszone geübten Praxis des „dissoziativen Föderalismus"106 konnte Frankreich aber immerhin eine Heraushebung der föderativen Elemente im Grundgesetz erreichen und auBerdem die Errichtung einer Sicherheitsbehörde initiieren ${ }^{107}$.

$\mathrm{Da}$ das deutschlandpolitische "double-bind“ der Franzosen - einem (Zentral)Staat zuzustimmen, ihn aber eigentlich nicht zu wollen - bestehen blieb ${ }^{108}$, gewann für sie der „Faktor Europa“ eine zunehmend wichtigere Rolle ${ }^{109}$. Das Bewußtsein von der wachsenden sowjetischen Gefahr hatte die Sorge vor Deutsch-

\footnotetext{
${ }^{77}$ Aufzeichnung Tarbé de Saint-Hardouin, 21. 10. 1948, zitiert nach: Wilkens, Besetzung, S. 16.

98 Ebd.

99 Vgl. Elgey, Histoire, Bd. 1, S. $479 \mathrm{f}$.

100 Erklärung Bidaults im Conseil des Ministres vom 26. 5. 1948, in: Auriol, Journal, Bd. 2, S. 242.

$101 \mathrm{Vgl}$. Wehner, Westalliierte, S. 412-427; zum Verlauf der parlamentarischen Auseinandersetzungen vgl. Kiersch, Parlament, S. 192-211.

102 Soutou, Place, S. 51.

$103 \mathrm{Vgl}$. Pfetsch, Verfassungspolitik.

104 S. Memorandum über den staatsrechtlichen Aufbau Deutschlands, 17.1. 1947, in: EA 1946/47, S. 623-626.

$105 \mathrm{Vgl}$. Wehner, Westalliierte, S. $223 \mathrm{f}$.

106 Pfetsch, Verfassungspolitik, S. 119.

$107 \mathrm{Vgl}$. Schlußkommuniqué der Londoner Sechs-Mächte-Konferenz, 7.6. 1948, nebst Anhang, in: EA 1948 , S. 1437-1439.

108 Kolboom, Frankreich, S. 74.

109 Poidevin, Faktor Europa; vgl. de Cuttoli-Uhel, Politique allemande, S. $107 \mathrm{f}$.
} 
land keineswegs völlig beseitigt. Folglich galt es, europäische Strukturen anzuregen, in die Westdeutschland einzubinden war. Wenige Tage vor seinem Abgang als Außenminister ergriff Bidault Mitte Juli 1948 die Initiative und machte auf einer Sitzung des Konsultativrates des Brüsseler Paktes den Vorschlag der Gründung einer „europäischen Versammlung“ und einer europäischen Wirtschafts- und Zollunion ${ }^{110}$.

Wenngleich er es also war, der das Umdenken in der französischen Deutschlandpolitik von der Dominanz zur europäischen Integration einleitete, verbindet die Geschichte diese Leistung mit einem anderen Namen. Bidaults Zustimmung zu den Londoner Vereinbarungen über die Weststaatsgründung hatte ihn bei Teilen des MRP so unbeliebt gemacht, daß er bei der Bildung der Regierung Marie am 24. 7. 1948 übergangen wurde. In die Historiographie ging daher sein Nachfolger Robert Schuman als jener Politiker ein, der die deutsch-französische Aussöhnung initiierte und vorantrieb.

Schuman stammte aus jenem Teil Lothringens, der zwischen 1871 und 1918 zum Deutschen Reich gehörte. 1886 im luxemburgischen Vorort Clausen geboren, studierte der deutsche Staatsbürger in Bonn, München, Berlin und Straßburg Rechtswissenschaften. Seit 1912 arbeitete er als Anwalt in Metz. Nachdem er mit der Rückkehr Elsaß-Lothringens einen französischen Paß erworben hatte, wirkte er von 1919 bis 1940 für den Parti Démocratique Populaire als Abgeordneter in der Assemblée Nationale. 1940 nach Deutschland deportiert, floh er 1942 und schloß sich der Résistance an. 1944 gehörte er mit Bidault zu den Gründungsmitgliedern des MRP, für den er 1946 in die Nationalversammlung zurückkehrte. Als Chef der Provisorischen Regierung holte Bidault ihn ins Kabinett, dem er bis Ende 1947 als Finanzminister angehörte, um dann für acht Monate selbst die Regierungsgeschäfte zu übernehmen. Mitte 1948 wechselte Schuman vom Matignon an den Quai d'Orsay und blieb dort, bis Bidault ihn im Januar 1953 beerbte. Nur noch einmal, 1955, saß er für elf Monate - als Justizminister - in der Regierung. Am Ende seiner politischen Laufbahn wirkte er von 1958 bis 1960 als Präsident, dann als Ehrenpräsident des Europäischen Parlaments ${ }^{111 .}$

Schuman war ein gemäßigter, katholischer Konservativer, entschlossen, das Trümmerfeld Europa so wieder aufzubauen, daß es zu demokratischer Stabilität fände. Seine - für französische Verhältnisse - lange Amtszeit im Quai d'Orsay, in der immerhin acht Regierungen wechselten, erlaubte es ihm, der Außenpolitik fünf Jahre lang seinen Stempel aufzudrücken. Wenngleich der spätere Mythos seines Verhältnisses zu Konrad Adenauer ebensowenig voll der Realität entspricht wie die Europa-Propaganda, die sein Bild als guten Europäer bestimmt, gehörte Schuman zu den wenigen französischen Politikern, die die Aussöhnung mit dem östlichen Nachbarn als ein zentrales Gebot ihrer Außenpolitik begriffen. Daß dem Grenzlandbewohner die deutsch-französische Verständigung schon in die Wiege gelegt worden sei, mag angesichts der innenpolitischen Rahmenbedingungen freilich eher bezweifelt werden ${ }^{112}$, und Schuman machte sie sich auch nicht so

110 Vgl. Dalloz, Georges Bidault, S. 300; Soutou, Georges Bidault, S. 290-292.

$111 \mathrm{Zu}$ Person und Politik Schumans vgl. Mittendorfer, Robert Schuman; Poidevin, Robert Schuman.

112 S. Hüser, „Doppelte Deutschlandpolitik“, S. 285 f. 
zur "Herzensangelegenheit" ${ }^{* 113}$, daß er die französischen Interessen dabei vergaß. So verfolgte er nach der Übernahme des Außenministeriums 1948 in der Demontagepolitik keineswegs eine konziliantere Linie als sein Vorgänger und setzte sich für die Fortsetzung der Dezentralisierung ein. Zugleich aber verteidigte er die Londoner Empfehlungen nach außen und hob im Dezember erstmals öffentlich auf einen europäischen Lösungsansatz des Ruhrproblems durch Zusammenlegung schwerindustrieller Ressourcen $a^{1{ }^{14}}$. Indem er umgehend versuchte, den europapolitischen Anstoß von Bidault umzusetzen und die Integration Westdeutschlands in Europa voranzutreiben, zielte er auch darauf $a b$, das geteilte Land vor nationalistischen Bestrebungen zu bewahren. Es sei notwendig, so schrieb Schuman im Oktober an René Massigli, „de presenter à l'imagination politique allemande un système continental où l'Allemagne ait sa part et son rôle“. Wenn die Westmächte nicht dazu in der Lage seien, der zukünftigen Regierung nicht nur Hoffnung, sondern auch einen Anfang europäischer Wirklichkeit zu geben, „le nationalisme allemand se cristallisera complètement autour de l'idée de restitution de l'unité allemande et l'Allemagne reprendra entre l'Est et l'Ouest un jeu de bascule adapté à cette fin " 115 .

Frankreichs Europaprojekt geriet indes bald ins Stocken, da England der anvisierten „europäischen Versammlung" keine Kompetenzen zugestand. So kam dieser erste europäisch-integrative Anlauf, der im Mai 1949 in den „Europarat" mündete, trotz französischen Insistierens auf der Schaffung einer „europäischen politischen Autorität mit begrenzten Funktionen, aber echten Vollmachten " über sehr beschränkte konsultative Möglichkeiten nicht hinaus ${ }^{116}$. Für eine feste Einbindung der entstehenden Bonner Republik konnte dies nicht ausreichen. Voller Mißtrauen verfolgte Paris die von Moskau ausgelöste Berlin-Krise, da sie die angelsächsischen Konzessionen gegenüber Deutschland zu steigern und die Entwicklung in Richtung auf einen zentralisierten deutschen Staat zu lenken drohte ${ }^{117}$.

Ohne jede Rücksichtnahme auf französische Interessen schienen die USA und Großbritannien von den Londoner Beschlüssen abzuweichen. Große Unruhe löste vor allem die angelsächsische Absicht aus, die Frage des Eigentums an den Ruhrbergwerken zugunsten der Deutschen zu entscheiden. Trotz energischer französischer Proteste übergaben die Oberbefehlshaber der Bizone ihnen mit der Verordnung Nr. 75 zur Reorganisation der Kohle- und Stahlindustrie der Ruhr Direktion und Geschäftsführung 118 provisorisch und überließen die Frage der endgültigen Besitzregelung der Entscheidung einer frei gewählten deutschen Regierung ${ }^{119}$. Immerhin aber einigten sich Frankreich, Großbritannien, die USA und

113 Lohse, Lockungen, S. 18.

114 Vgl. Hüser, „Doppelte Deutschlandpolitik“, S. 282-297.

115 Schuman an Massigli, 7. 10. 1948, zitiert nach: Poidevin, Robert Schuman, S. 221.

116 Empfehlungen des Europarates an die Versammlung, September 1949, in: EA 1949, S. 2559; vgl. Loth, Weg, S. 69-78.

117 Vgl. Fritsch-Bournazel, Mourir?, S. $172 \mathrm{f}$.

118 S. "Gesetz Nr. 75 über die Umgestaltung des deutschen Kohlebergbaus und der deutschen Eisenund Stahlindustrie" für das amerikanische und britische Kontrollgebiet, 10.11. 1948, in: Gesetzblatt der Verwaltung des Vereinigten Wirtschaftsgebietes, 1947 bis 1949, Beilage Nr. 1 zum Gesetzblatt der Verwaltung des Vereinigten Wirtschaftsgebietes, 1949, S. 1-15.

119 Vgl. Poidevin, Frankreich und die Ruhrfrage, S. 325 f.; Wehner, Westalliierte, S. 428-434. 
die BENELUX-Staaten am 28. 12. 1948 auf die Einrichtung einer Internationalen Ruhrbehörde und eines Militärischen Sicherheitsamtes zur Kontrolle der Montanindustrie und der Demilitarisierung ${ }^{120}$. Dennoch empfand man die Entwicklung an der Seine als „Zusammenbruch“ der eigenen deutschlandpolitischen Positionen. Der Leiter der Unterabteilung Zentraleuropa im Quai d'Orsay Pierre de Leusse empfahl Schuman daher, auf die Einhaltung der alliierten Vereinbarungen zu drängen und die Organisation Europas als „dérivatif puissant aux aspirations allemandes" zu forcieren, indem allen interessierten Staaten Europas unter Einschluß der Länder Westdeutschlands der Beitritt gewährt werden sollte. Als letztes dramatisches Mittel erwog er den vollständigen Rückzug aus Deutschland, um nicht in die Wiederherstellung der deutschen Macht verwickelt zu werden ${ }^{121}$.

Eine solche Empfehlung läßt sich natürlich kaum mit der mitunter in der Literatur vertretenen These in Einklang bringen, Frankreich habe sich „nicht erst unter dem Druck anglo-amerikanischer Pressionen zu einem Wandel seiner Deutschlandpolitik“ entschlossen ${ }^{122}$. Die weiter wachsende Einsicht in die unhaltbaren eigenen Positionen verstärkte die französische Umorientierung. Als auch alliierte Stellen die Bereitschaft zur Anhebung der 1947 auf 11 Millionen Tonnen festgelegten Begrenzung der jährlichen deutschen Stahlproduktion signalisierten, schien de Leusse nur noch eine freiwillige europäische Eingliederung der deutschen Wirtschaft als Ausweg möglich: „Nur durch einen freiwilligen Verzicht auf einen gewissen Teil der Souveränität der Staaten, nur durch eine Assoziierung der Interessen dieser Staaten kann sich Frankreich, indem es Deutschland auf diese Weise bindet, die Garantien seiner Sicherheit verschaffen, die es benötigt." 123 Die deutsch-französische Auseinandersetzung wurde für „beendet“ erklärt und durch eine neue Tatsache ersetzt, „nämlich die der schicksalhaften Gemeinschaft von Franzosen und Deutschen“. Demgemäß galt „die Rückkehr Deutschlands in das Konzert der europäischen Mächte als [ein] in politischer, wirtschaftlicher und sozialer Hinsicht gleichberechtigter Partner [als] unausweichlich"124. Die Zeit drängte, denn die politische Organisation Europas mußte vorbereitet sein, bevor Westdeutschland seine Regierung gebildet hatte.

Der „début de réalisation européenne" 125 wurde indes von der französischen Diplomatie keineswegs als "Allheilmittel“ gegen die „deutsche Gefahr" begriffen. Schon im Januar 1949 warnte der ansonsten die Einbindung Deutschlands in die europäische Union wärmstens befürwortende de Leusse, eine solche Union beschwöre die Gefahr, daß Deutschland eine Stellung einnehmen könne, „die ihm die Hegemonie verschafft, nach der es immer getrachtet hat“. Als Gegengewicht empfahl er daher eine enge Assoziation mit England innerhalb der Union ${ }^{126}$. Par-

120 Vgl. ebd., S. 434-497.

${ }_{121}$ Aufzeichnung de Leusse, 24. 11. 1948, zitiert nach: Wilkens, Besetzung, S. 19; s. a. Poidevin, Faktor Europa, S. 414; ders., La France devant le danger allemand, S. 262.

122 Hudemann, Weichenstellungen, S. 138.

${ }^{123}$ Aufzeichnung de Leusse, 4. 1. 1949, zitiert nach: Wilkens, Besetzung, S. 20; s. a. Hitchcock, France Restored, S. 108-110.

${ }^{124}$ Aufzeichnung der Abt. Europa im MAE, 14. 7. 1948, im Auszug abgedruckt in: Knipping, „Que faire?", S. 153 f., hier S. 154 u. 153.

125 Schuman an Massigli, 7. 10. 1948, zitiert nach: Poidevin, Robert Schuman, S. 221.

126 Aufzeichnung de Leusse, 4. 1. 1949, zitiert nach: Poidevin, Faktor Europa, S. 411. 
allel bemühte sich die französische Regierung daher, die Entwicklung jenseits des Rheins durch eine Reihe einseitiger Restriktionen unter Kontrolle zu halten. So sollte das Provisorische der Neugründung unterstrichen werden, das Gewicht der Länder möglichst hoch sein, eine Bewaffnung ausgeschlossen bleiben und die Internationale Ruhrbehörde möglichst große Kompetenzen erhalten.

Ein Teil dieser Forderungen konnte umgesetzt werden. Auf der Washingtoner Konferenz fixierten die drei westlichen Außenminister am 8. 4. 1949 nicht nur ein Ruhrstatut, das die Verteilung der Erträge, die Dekartellisierung und Investitionslenkung bei ihnen beließ, sondern verabschiedeten auch ein Besatzungsstatut, das den Provisoriumscharakter, die Beschränkung der Demontagen und die dauernde Entmilitarisierung der entstehenden Bundesrepublik betonte. Die entscheidende Frage der Verfügungsgewalt über die Ruhrindustrie wurde jedoch nicht im französischen Sinne beantwortet ${ }^{127}$.

Darüber hinaus bemühte sich Frankreich, sein Sicherheitsbedürfnis durch den Aufbau eines Militärbündnisses mit den USA und den westeuropäischen Staaten zu befriedigen. Die Verhandlungen über die Struktur der Nordatlantischen Allianz wurden dabei stets eng mit jenen über den Status Westdeutschlands verknüpft, wie die Erklärung des Berichterstatters des Auswärtigen Ausschusses der Assemblée Nationale René Mayer am 22. 7. im Palais Bourbon unterstreicht: Dieser Pakt „est dirigé contre toute agression qu'elle soit, et je crois avoir dit ailleurs [...] qu'il pourrait être dirigé contre l'Allemagne si les erreurs des anciens adversaires de l'Allemagne [...] la remettaient un jour en position d'attaquer ses voisins occidentaux." 128

Die sich nun abzeichnende Lösung der „deutschen Frage“ blieb gewiß hinter den ursprünglichen französischen Vorstellungen zurück, zumal sie einen weitgehenden Verzicht auf eine eigenständige Großmachtpolitik erforderte. Hinsichtlich der Saar konnte Frankreich die wirtschaftliche Angliederung, in der Ruhrfrage nur eine lockere Kontrolle erreichen ${ }^{129}$. Den Wiederaufbau des wirtschaftlichen Potentials des Ruhrgebietes vermochte es nicht zu vereiteln und auch keine Gebietsabtretungen in Westdeutschland durchzusetzen. Die Bilanz war „sehr mager" 130 , aber akzeptabel, da sie den französischen Sicherheitsbedürfnissen Rechnung trug und die Westintegration der Bundesrepublik in Form und Umfang auf französische Initiativen zurückging.

127 Vgl. Hitchcock, France Restored, S. 110f.; Milward, Reconstruction, S. 160-164.

128 Erklärung Mayers in der Assemblée Nationale, 22. 7. 1949, in: Annales de l'Assemblée Nationale, Débats 1949, Bd. 7, S. 5064-5070, hier S. 5068.

129 Vgl. Kerkhoff, Großbritannien, S. 76-81; Pohlmann, Saarfrage, S. 209-220.

130 Poidevin, Frankreich und die Deutsche Frage, S. 420. 